\title{
Modeling Information Transparency of Economic Entities under Uncertainty
}

\author{
Hanna Kucherova* \\ Economics Department \\ Classic Private University \\ Zaporizhzhia, Ukraine \\ kucherovahanna@gmail.com
}

\author{
Anastasiia Didenko \\ Economics Department \\ Classic Private University \\ Zaporizhzhia, Ukraine \\ Didenko.naya@gmail.com
}

\author{
Olena Kravets \\ Economics Department \\ Classic Private University \\ Zaporizhzhia, Ukraine \\ Eva_84@i.ua
}

\begin{abstract}
Topicality of modeling information transparency is determined by the influence it has on the effectiveness of management decisions made by an economic entity in the context of uncertainty and information asymmetry. It has been found that information transparency is a poorly structured category which acts as a qualitative characteristic of information and at certain levels forms an additional spectrum of properties of the information that has been adequately perceived or processed. As a result of structuring knowledge about the factor environment, a cognitive model of information transparency was constructed in the form of a weighted digraph. Structural analysis of the cognitive model made it possible to evaluate the classes of factors, identify their limited relations, establish the centrality of the roles of information transparency and information and communication security in the system built and evaluate their importance when modeling the situation self-development. Information visibility has been found to have the strongest impact on the system. Taking into account different initial weights of the key factors information transparency and information and communication security - the study substantiates the strategic ways for economic entities to achieve their goals in the context of uncertainty and information asymmetry, which allows us to use this approach as a tool for strategic management in the information environment.
\end{abstract}

Keywords-information transparency, cognitive modeling, digraph, factors, relations, strategic management.

\section{INTRODUCTION}

Information transparency is a possibility for any economic stakeholders to track the chain of actions and stages of forming the information content [1] which is important enough to make effective management decisions. On the one hand, information transparency in required scope ensures that its provider can be held accountable for the content and consequences of its disclosure, while on the other it builds trust and reliance on a company and reduces alienation between interaction parties [2]. That is, systemic information transparency determines positive expectations regarding company development, builds up confidence and improves business climate, which is always relevant in the context of competition, economic downturn, changes in socio-political and socio-economic vectors of development.

From the point of view of content understanding, information transparency is interpreted by authors [3] as a requirement, norm, standard or as a goal which, being set, defines the specifics of achieving it. Thus, transparency as one of the 8 major characteristics of the Good governance concept [6] means that decisions taken and their implementation follow rules and regulations. It also means that information is freely available and directly accessible to those who are affected by the decisions and their enforcement. According to the concept, main attributes of information are the scope of information provided and that it is provided in easily understandable forms and media. Another approach can be found in the article whose authors define transparency as perceived quality of the information intentionally shared by its sender [8]. We believe that information transparency is a poorly structured category which nevertheless is a qualitative characteristic of information and at certain levels forms an additional spectrum of properties of the information which has been adequately perceived or processed. Ambiguity of information transparency complicates drawing its overall portrait for comprehensive research and identifying its optimal level for different economic entities and their various purposes.

Furthermore, in economics poorly structured categories are generally studied by means of soft modeling including dynamic cognitive modeling and cognitive mapping. In our case, information transparency is the object of the research, while cognitive modeling of how transparency and its constructs influence each other is its subject. Scientific search is aimed at structuring the information transparency factor environment, identification of strategic changes in its level and the levels of its factors which result in accelerated and decelerated development of the system, extracted from a specific eco-socio-economic environment during $\mathrm{t}$ period. Information type, degree of completeness of information, the type of the company's operations, its size, organizational and legal form are decisive factors for characterizing its information transparency. Thus, for economic entities information can be transparent, partially transparent or nontransparent. The main parameters of information transparency management are time, tactical, strategic and technological constrains on the flow of information for a limited or unlimited number of stakeholders. Information flow in the research [3] is characterized by the following parameter tuple: $<\mathrm{I}, \mathrm{IP}, \mathrm{IR}, \mathrm{IM}, \mathrm{IE}>$, information (I), information provider (IP), information receiver (IR), information medium (IM), and information entity (IE). It seems reasonable to add information constraints (such as on the scope of information usage) to this set of defined parameters. It is also appropriate to take into account the period with its inherent social and economic trends, which can ensure more accurate and adequate interpretation of results. The authors [3] place emphasis on the importance of 
subject affiliation when studying the risks of achieving information transparency, which explains different quality of certain level of information transparency for different stakeholders. Information receivers and media also differ in terms of their goals, capabilities and time allocated to the operation, which should also be taken into consideration when assessing the feasibility of increasing or reducing information transparency in order to improve rather than aggravate the situation.

\section{STRUCTURING INFORMATION ON FACTOR LOADING}

A. Attributes of information transparency - visibility, risk, availability, technologies.

One of the main attributes of information transparency is visibility of information content which in its turn is determined by general availability of information, accessibility of information to third parties and degree of approval to disseminate information [1]. Information visibility is also differentiated according to its degree as visible, partially visible and invisible. In their article [1] the authors argue that high information visibility may result in attention span, produce a flood of information, overwhelming our cognitive and interpretive capabilities, thus rendering the information meaningless or confusing. Moreover, maximum visibility can mask formality of actions or results of the decisions taken, which are presented in the information [1]. On the other hand, insufficient disclosure of information may result in an attempt to compensate for it with some other, forecasted, predicted, guessed, insider information, trustworthiness and reliability of which may not be sufficient to make an effective management decision. The authors of the article [2] point out that transparency arises under the condition of certain balance between the degree of information disclosure and the degree of its perception and understanding by users. Hence, we can make a conclusion that only limited (up to a certain level) visibility ensures high level of transparency, provided that it is the key and quality information that is disclosed, not its noises. In general, the degree of information transparency determines the associated risk of its disclosure or concealment. According to the article [2], information visibility is determined by how responsible its providers are, that is, how consciously they assess the advantages, necessity and need to disclose certain amout and type $f$ information. The attitude and policy of an economic entity in terms of establishing the boundaries of information transparency determine the degree of confidence it enjoys as an information provider and medium. Information availability depends on how well information reflects actions and decision-making stages in easily recognizable data forms through a reliable storage of those data. In the article [1] it is interpreted as a degree of complexity associated with the extraction and interpretation of information. It is determined by such attributes as possibility for an unlimited number of people to be informed, insignificant amount of time, finances and efforts necessary to find the relevant information, veracious perception of information content. Information availability is ensured by its relevant classifiers, systematizers etc. Ongoing development of information and communication technologies has been improving the conditions, forms and methods of storing and transmitting information as accurately as possible, which allows us to expand the possibilities of managing information transparency and its constructs. In order to assess the level of information and communication technologies development, different indices are used, including ICT Development Index, Networked Readiness Index (surveys at the state, business and community levels). Growing complexity of information and communication technologies requires continuous improvement of existing skills on the part of economic entities and drives the need for life-long learning. At the same time, technological advances mean higher associated risks, which urges stakeholders to provide the required level of information and communication security.

\section{B. Attributes of information transparency - cognitive limitations, efficiency, corruption}

Adequate and realistic perception of information content by receivers, which is crucial for effective decision making, is determined by cognitive limitations of economic entities. Information overload may result in deliberate distancing from all information sources. In addition, dubious quality of information, its shortage or poor relevance cause wrong decisions. We cannot ignore the human factor, i.e. the difference in perception of the same facts by different economic entities, which also contributes to information asymmetry. In general, the above determines how effectively an economic entity uses the information visible to it. Information society has been creating new information services and changing approaches to delivering old ones by means of increasing use of modern technologies. Therefore, in order to understand the state of implementing information and communication technologies in society and evaluate the effectiveness of new technologies introduction, there is an urgent need to develop approaches to evaluate knowledge and skills of the service receivers, their social communication skills and skills large-scale data processing [15].

When discussing information transparency, it is absolutely necessary to take into account the role of transparency as an economic entity's accountability. It is not the information itself that is considered, but rather the potential disclosure of that information, which forces stakeholders to "do the right thing", so information transparency is often quite closely linked to the problem of corruption. Hence, we think of information transparency as a vital tool that helps reduce corruption [4].

We find the list of information transparency factors, suggested by the experts, substantial, but offer to add information transparency levels, degrees, and constraints that occur in a real-life environment when it transfers from one state to another. This will help to substantiate the feasibility of achieving a change in specific degrees or levels of factors, informational transparency, in order for stakeholders to achieve their goals. As a result of structuring knowledge on factor loading of information transparency we have identified the following main factors that accelerate and decelerate the development of an economic entity system: the degree of confidence in the medium and source of information, the degree of the economic entity responsibility, the scope of information visibility, the degree of information availability, the degree of information accessibility to third parties, approval to disseminate information, the degree of information reliability, the level of information and communication technologies development, cognitive limitations of the user, the level of 
information use efficiency, the level of information and communication security, the level of corruption reduction, risks of information disclosure or concealment.

\section{METHODS}

The methodology of information transparency evaluation includes a wide scope of modern methods, from social research to modeling; results of many surveys and observations are studied using econometric tools. The results of critical analysis presented in the article [16] show that there are different approaches to transparency evaluation (market-based, analyst perception-based and accountingbased measures) and researchers invoke different techniques including web content analysis, verbal protocol study, principal component analysis etc. However, it is index approach that has gained popularity in this area of research. In addition, information transparency is defined as a component of a more general indicator, or examined in the context of information type (financial, fiscal, political, economic information). The index method was used in the study [4] to measure Information Transparency Index and the Accountability Transparency Index constructed using a methodology similar to the one used in Transparency International's Corruption Perceptions Index. In the first stage, the researchers selected indicators that determine the index for the maximum period, and normalized them. The resulting index was calculated as a mean of the components of its normalized indicators. The large-scale study [9] provides a transparency index for 194 countries. The indices comprise an aggregate transparency index with two subcomponents: economic/institutional transparency and political transparency. By means of correlation and regression analysis using substantiated indicators, the authors showed that information transparency is associated with better human and economic development indicators, higher competition and lower corruption. Political transparency has also been studied separately, the main indicator used over the years has been the Freedom House Freedom of the Press index. Its advantage is extensive coverage across countries combined with significant temporal coverage (going back to 1979). [4]. Economic transparency index was presented in the article [10] where it was developed using regression estimation (based on the World Bank and IMF data). The index measures the frequency with which governments update economic data that they make available to the public as a condition of existence (or absence) of laws on freedom of information. The authors took into account the quantity of data released by governments, rather than their timeliness. As a result, they proved that information transparency is directly proportional to effective governance. It is also worth mentioning the Open Budget Index, prepared by the International Budget Partnership, which is aimed at studying fiscal transparency [4]. In addition to these indicators, transparency is studied as a component within larger frameworks. In particular, the World Bank's CPIA indicators have a component on "transparency in the public sector". Information transparency for businesses in terms of obtaining information about government policies and regulations affecting business activities is considered in the Global Competitiveness Report, while freedom of the press as a condition for information transparency is represented in the CIRI Human Rights database [4]. The research [11] offers to use a modified Delphi technique in order to study information transparency. This technique implies having rapid reviews from stakeholders, namely researchers, policy makers, industry and health care providers. However, the results of this approach are rather subjective as respondents often change their opinion under the influence of different factors. The paper [12] proposes four reference models which form a baseline for transparency requirements in information systems. The models cover actors involved in the process of ensuring transparency and information circulation among them, transparency meaningfulness, transparency usefulness for a specific audience, and information quality in transparency. The reference models are further used to create TranspLan, a modeling language for capturing and analyzing information transparency requirements among stakeholders [13]. Thus, a broad range of methodological tools gives researchers quantitative measures of information transparency, the impact it makes and gets. However, each approach or method has inherent risks when used in practice due to assumptions, limitations that are not achievable in real life. In addition, there is no general system of forming information transparency as an economic category since it is impossible to formalize this poorly structured category without considerable impact of subjectivity and assumptions. Considering the importance of the cognitive method when studying poorly structured categories, we propose to analyze information transparency using the methodology of cognitive modeling and impulse processing [17]. The proposed approach is based on structuring knowledge about the object of study, building a cognitive model of object development in the form of a digraph, scenario modeling of the dynamics in the state of the system when it is stable and changing, justification of the option that can help to achieve the goals of the study. Suppose a signed digraph with adjacency matrix $A$ determines information transparency and its factor environment. The vertices of the digraph are represented by the set $u_{1}, u_{2}, \ldots, u_{n}$. Every vertex $u_{i}$ has the value of $v_{i}(t)$ at discrete moments of time $t=0,1,2, \ldots$. The value of $v_{i}(t+1)$ is determined by the value of $v_{i}(t)$ and information on whether other vertices $u_{j}$, adjacent with $u_{i}$, have increased or decreased their values at moment $t$. The change of $p_{j}(t)$, set by the difference of $v_{j}(t)-v_{j}(t)$, is called a pulse if $t>$ 0 . The initial condition should be specified when $t=0$. We introduce the following notation:

$$
\operatorname{sgn}\left(u_{j}, u_{i}\right)=\left\{\begin{array}{l}
1, \text { if edge is positive }, \\
-1, \text { if edge is negaive }, \\
0, \text { if edge is missing } .
\end{array}\right.
$$

Then for $t \geq 0$ we can find:

$$
v_{i}(t+1)=v_{i}(t)+\sum_{j=1}^{n} \operatorname{sgn}\left(u_{j}, u_{i}\right) p_{j}(t)
$$

Modern information technologies provide researchers with a possibility to simplify cognition procedure. In particular, to achieve the goal of this research we can use some software products, such as Decision Explorer Application, aimed at providing the user with illustrative cognitive maps which can be further analyzed to better understand the issue under study, links between its factors. 
Another specialized software product is FCMapper which helps to analyze, model fuzzy cognitive maps, explore behavior of the system and interaction between its factors. FCMapper calculates structural characteristics of the system based on the created digraph, and provides a variety of scenarios. The logistic squashing function is used for standard scenario calculation. It can be written as $f(x)=1 /\left(1+e^{-x}\right)$ [18]. The results obtained for each scenario are compared with the initial one, where the output weights for each vertex are set at the same level, that is, the situation self-development is modeled. The final development of the system in the form of a cognitive model in the absence of external influences can be reduced to a fixed-point attractor, a boundary cycle of repeating binary vectors, or to chaotic or aperiodic attractors. [19].

\section{RESUlTS}

As a result of structuring knowledge on factor environment of information transparency we have identified a set of vertices and their cause-effect relations; together they determine the corresponding cognitive model in the form of a weighted digraph (Figure 1). For the constructed digraph, based on the situation simulation, the following vertices have been found to have the greatest influence on target vertex 1: 8, 9, 11, 12, 14 .

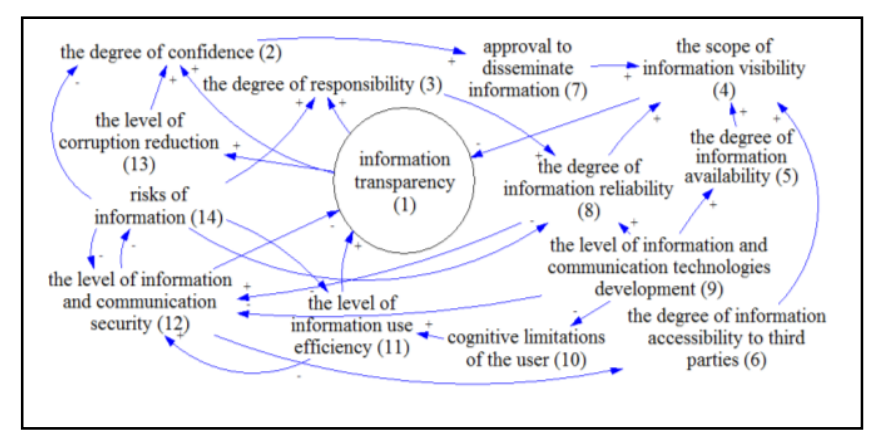

Fig. 1. Cognitive model of information transparency

However, not all of these vertices can be affected in terms of goals and opportunities of economic entities. The following figure shows the results of studying a simulation of the vertex parameter dynamics in the context of system interaction without external influence for $t=12$, where $t$ is the study period, and the initial values of the vertex parameters $\mathrm{v}_{\mathrm{i}}=0$ (Figure 2).

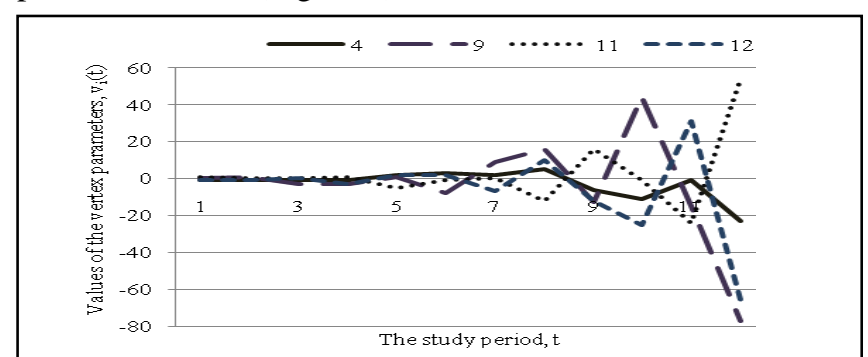

Fig. 2. The dynamics of the value of target vertex 1 under the influence of vertices $4,9,11,12$

The diagram shows the dynamics of the influence that vertices $4,9,11,12$ have on the target vertex (1). The diagram shows variable values of vertex 1 , but we can see that factors 12 and 4 tend to have a negative influence, factor 11 has a positive influence, while factor 9 does not have a clearly defined tendency during the period under study. Let us now examine the effect of changes in transparency level on vertices 2, 3, 7 and 13 (Figure 3).

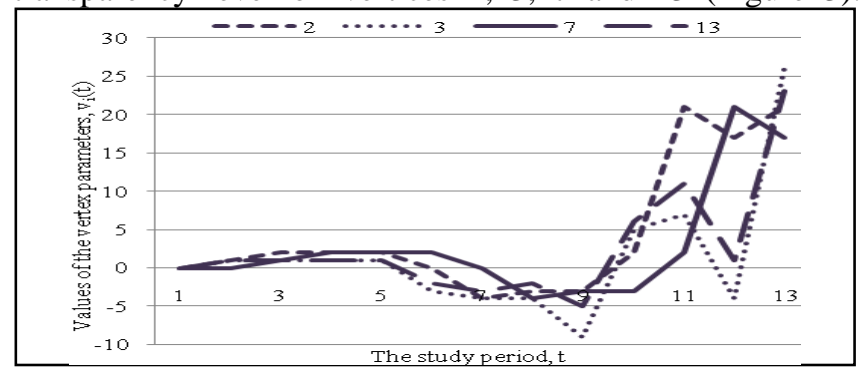

Fig. 3. The dynamics of the influence of target vertex 1 on vertices $2,3,7$, 13.

We can see a positive dynamics of the influence that target vertex 1 had on vertices 2, 3, 7 and 13 throughout the period under study. Having analyzed structural characteristics of the cognitive map using FCMapper, we arrived at the following results. One vertex functions as a Transmitter, it is vertex 9, the level of technology development. Other vertices are Ordinary, there are no Receiver vertices in the model. Classification of the cognitive model vertices by these classes helps us better understand the structure of the graph. Having analyzed the cognitive model using the Decision Explorer Application, we identified two key elements of the cause-effect relationship system (based on the calculation of vertices centrality which reflects the strength of relationships between the vertices). The index that shows the proportion of existing connections of potentially possible (density) is calculated to be 0,14 . It gets values within $[0 ; 1]$. The higher its value is, the more active interaction between the vertices takes place. They are the information and communication security (12) and information transparency (1). Using FCMapper software you can set the initial weights of vertices and investigate their influence on other system indicators. To quantify the system development dynamics, the influence of one vertex on the other is represented by the following set of values: very strong $(0.8 ; 1]$; strong $(0.6$; $0.8]$; medium $(0.4 ; 0.6]$; weak $(0.2 ; 0.4]$; and very weak $(0$; 0.2]. During the situation self-development modeling (the total number of iterations is 60) the constructed model came to a stable state, which was achieved due to feedbacks, with the maximum number of iterations (51) for the degree of information accessibility to third parties (6) and risks (14), the fewest iterations (25) were required for the availability (5) and technology development (9) vertices. The calculations obtained indicate that the factor with the greatest impact on the system is the scope of information visibility (4). Information reliability (8) and approval to disseminate information (7) are found to be of high influence. The degrees of confidence (2), responsibility (3) and information availability (5) also showed significant influence. Then we analyzed the situation development scenarios under the change of the key elements of the causeeffect relationship system, namely the level of information and communication security (12) and information transparency (1). For the first scenario the initial vertex value was set at a low level $(0.1)$, for the second scenario at a medium level (0.5) and for the third one at a high level (0.9). When the value of vertex 12 is of low and medium level, the most positive changes are observed for the 
following vertices: information transparency (1), the degree of responsibility (3), the degree of information accessibility to third parties (6), the level of corruption reduction (13) and risks (14). The high value of vertex 12 has the most positive influence on the level of information use efficiency (11). When the value of vertex 12 is of low and medium level, the most negative changes are observed for such vertices as the degree of information reliability (8) and the level of information use efficiency (11) The high value of vertex 12 has the most negative effect on the vertices of information transparency (1), the degree of responsibility (3), the degree of information accessibility to third parties (6), and risks (14). Now, we can have a close look at the influence of information transparency (1) on the cognitive model factors. In general, the level of information transparency does not affect the system significantly, it is 0.32 in the context of the system self-development scenario. At the same time, a decrease in the level of vertex 1 reduces the influence of such factors as the degree of responsibility (3) and the degree of confidence (2) on the system. An increase in the level of vertex 1 results in the growing influence of the corruption reduction factor (13), while low level of vertex 1 causes an increase in the level of corruption (13). A low level of vertex 1 slightly increases such factors as risks (14) and information accessibility to third parties (6), The value of the approval to disseminate information (7) increases when the level of vertex 1 is higher.

\section{CONCLUSIONS}

It involves structuring knowledge about the factor environment, identifying strategic changes in the level of information transparency and the levels of influence of its factors. Information transparency is defined as a poorly structured category which nevertheless acts as a qualitative characteristic of information, a certain level of which forms an additional spectrum of properties of information which has been adequately perceived or processed. In the course of the study, a cognitive model of information transparency was constructed in the form of a weighted digraph. The results of its structural analysis revealed that the degree of transparency and information and communication security have the most powerful influence on the state of the system. The results showed that higher levels of information and communication security lead to lower risks, lower degree of information accessibility to third parties and information transparency in general. At the same time, only the high level of information and communication security is associated with an increase in the degree of information reliability, the level of visible information use efficiency and information transparency. The analysis of the cognitive model factors which affect the level of information transparency showed that its level is most significantly decreased by the growing level of information and communication security, while it is most significantly increased by the growing efficiency of visible information use, higher level of technological development and reduced scope of information visibility. The results of this study allow us to identify the strategic elements of managing information transparency as a tool for economic entities to achieve their goals in the information environment.

\section{REFERENCES}

[1] C. Stohl, M. Stohl, and P. Leonard, "Managing Opacity: Information
Visibility and the Paradox of Transparency in the Digital Age", International Journal of Communication, vol. 10, pp. 123-137, 2016.

[2] K. Kundeliene, and S. Leitoniene, "Business Information Transparency: Causes and Evaluation Possibilities, Procedia", Social and Behavioral Sciences, vol. 213, pp. 340-344, 2015. [Online]. Available: http: http://www.sciencedirect.com/science/article/pii/ S1877042815059030. Accessed on: August 5, 2019.

[3] M. Hosseini, A. Shahri, K. Phalp, and R. Ali, "Tansparency as a Requirement" in Proc. 21st REFSQ Conference. Poster and Demo Track, Bournemouth University, pp. 222-223, 2015. [Online]. Available: http://ceur-ws.org/Vol-1342/03-Posters.pdf. Accessed on: August 7, 2019.

[4] A. Williams, "A global index of information transparency and accountability", Journal of Comparative Economics, vol. 43, pp. 804824, 2014. [Online]. Available: http://dx.doi.org/10.1016/j.jce. 2014.10.004. Accessed on: August 3, 2019.

[5] R. Bushman, and A. Smith. "Transparency, financial accounting information, and corporate governance." SSRN Electronic Journal, vol. 9, pp. 65-87, 2003.

[6] Y. Sheng, "What is Good Governance?", United Nations Economic and Social Commission for Asia and the Pacific, 2009. [Online]. Available: www.unescap.org/sites/default/files/good-governance.pdf. Accessed on: August 1, 2019.

[7] A. Vaccaro, "Privacy, security, and transparency: ICT-related ethical perspectives and contrasts in contemporary firms", Social inclusion: Societal and organizational implications for information systems, vol. 208, pp. 245-258, 2006

[8] A. Schnackenberg, and E. Tomlinson, "Organizational transparency: A new perspective on managing trust in organization-stakeholder relationships", Journal of Management, vol. 42.7, pp. 1784-1810, 2016.

[9] A. Bellver, and D. Kaufmann, "Transparenting transparency: initial empirics and policy applications", 2005. [Online]. Available: http://ssrn.com/abstract=808664. Accessed on: August 7, 2019.

[10] R. Islam, "Does more transparency go along with better governance?", Economics and Politics, vol. 18 (2), pp. 121-167, 2006.

[11] J. Knottnerus, "Promoting transparency of research and data needs much more attention", Journal of Clinical Epidemiology, vol. 70, pp. 1-3, 2016. [Online]. Available: https://www.jclinepi.com/article/ S0895-4356(16)00015-9/pdf. Accessed on: August 1, 2019.

[12] M. Hosseini, A. Shahri, and K. Phalp, "Four reference models for transparency requirements in information systems", Requirements Engineering, vol. 23 , no. 2, pp. 251-275, 2018. [Online]. Available: https://doi.org/10.1007/s00766-017-0265-y. Accessed on: August 3, 2019.

[13] M. Hosseini, A. Shahri, and K. Phalp. "A modelling language for transparency requirements in business information systems" in Proc. of the 28th international conference on advanced information systems engineering (CAiSE), Springer, 2016, pp. 239-254.

[14] A. Florini et al., "Does the invisible hand need a transparent glove? The politics of transparency", in Proc. of the world banks annual conference on development economics, Washington, 2000, pp. 163184.

[15] Measuring the Information Society Report 2018. Volume 1. (2018, December 10-12). [Online]. Available: https://www.itu.int/en/ITUD/Statistics/Pages/publications/misr2018.aspx

[16] K. Kundeliene, and S. Leitoniene, "Business Information Transparency: Causes and Evaluation Possibilities, Procedia", Social and Behavioral Sciences, vol. 213, pp. 340-344, 2015. [Online]. Available: http: http://www.sciencedirect.com/science/article/pii/S18 77042815059030. Accessed on: August 5, 2019.

[17] F. Roberts, Discrete mathematical models, with applications to social, biological, and environmental problems. Englewood Cliffs, N.J.: Prentice-Hall, 1976.

[18] FCMappers, 2019. [Online]. Available: http://www.fcmappers.net. Accessed on: August 1, 2019.

[19] O. Osoba, and B. Kosko, "Fuzzy cognitive maps of public support for insurgency and terrorism", Journal of Defense Modeling and Simulation: Applications, Methodology, Technology. no. 14 (1), pp. 17-32, 2017.

[20] Y. K. Sheng, “What is Good Governance?", United Nations Economic and Social Commission for Asia and the Pacific. [Online]. Available: www.unescap.org/sites/default/files/good-governance.pdf. Accessed on: August 1, 2019. 